\title{
ELEKTRA: Um Chatterbot para Uso em Ambiente Educacional
}

\author{
Michelle Denise Leonhardt* \\ Daiane Dorneles de Castro ** \\ Renato Luís de Souza Dutra *** \\ Liane Margarida Rockenbach Tarouco ${ }^{* * * *}$
}

\begin{abstract}
Resumo. A Educação à Distância vem despertando muito interesse de pesquisadores de diversas áreas. Por isso, também vem sendo muito abordada em congressos, workshops e seminários. Muito se discute sobre a união entre tecnologia e educação visando complementar a formação de estudantes. Com a pesquisa em Inteligência Artificial, surgiram os chatterbots. Chatterbots são programas destinados a demonstrar a capacidade racional de um computador, ou seja, buscam imitar o comportamento da inteligência humana. Este artigo apresenta uma implementação de um chatterbot educacional para o ensino de física e redes de computadores, a Prof ${ }^{a}$ Elektra, baseado no ALICE, um chatterbot bastante utilizado no mundo
\end{abstract}

Palavras-chave: Chatterbots, AIML, Educação à Distância, Redes de Computadores

Abstract. Distance learning has been raising the interest of researchers of several different fields. Due to this fact, it has been the discussion topic of many congresses, workshops and seminars. Much is discussed about the use of both technology and education to complement the capabilities of students. With the research of Artificial Intelligence, chatterbots appeared. Chatterbots are computer programs, which are used to demonstrate the rational capacity of a computer. They intend to imitate the behavior of human intelligence. This article presents a implementation of a educational Chatterbo for Physics and Computer Networks teaching, Professor Elektra, which is based in ALICE, a chatterbot used around the world.

Key words: Chatterbots, AIML, Distance Education, Computer Networks

\footnotetext{
* Mestranda em Ciência da Computação pela UFRGS, mdleonhardt @inf.ufrgs.br

** Graduanda em Sistemas de Informações pela PUCRS, bolsista do projeto METROPOA, daiane@ cinted.ufrgs.br

**** Doutorando em Informática na Educação pelo PGIE-UFRGS, Mestre em Ciência da Computaçào pela PPGC-UFRGS, rdutra@pgie.ufrgs.br

${ }^{* * * *}$ Doutora em Engenharia Elétrica/Sistemas Digitais pela USP, Coordenadora do CINTED-UFRGS, Professora do PGIE-UFRGS, liane@penta.ufrgs.br
} 


\section{Introdução}

"Podem as máquinas pensar?" Esta pergunta, proposta no texto do matemático Alan Turing: Computing, Machinery and Intelligence, publicado originalmente em 1950 originou um teste que chamou de Jogo da Imitação, mas que veio a ser conhecido como o Teste de Turing. Na sistemática do jogo, um interrogador, comunicando-se via terminal com um software e, simultaneamente, com uma outra pessoa, deveria descobrir quem era quem. O matemático Alan Turing morreu em 1954, uma década antes de programas que simulam o diálogo humano começarem a proliferar.

Há muito tempo o ser humano procura desenvolver a comunicação natural entre interlocutores. Com o avanço da tecnologia e, conseqüentemente, a popularização de acesso aos computadores pessoais e à internet, essa preocupação também foi sendo transferida para as máquinas, onde a possibilidade de se dar a uma máquina habilidade para interagir com o ser humano, através de uma compreensão e simulação do seu comportamento, tem sido, alvo de pesquisas na área de Inteligência Artificial. A inteligência artificial desenvolveu ao longo dos anos diversas maneiras de simular a comunicação humana, acarretando, entre outras tecnologias, o surgimento dos chamados chatterbots, que são programas de computador que simulam uma conversa com uma pessoa.

Nesse contexto, foram surgindo diversas categorias de chatterbots utilizadas na internet. Os chatterbots para fins educacionais demonstram-se interessantes, pois permitem que alunos se relacionem de forma mais humana com o computador. Robôs de conversação oferecem muitas vantagens em um ambiente de aprendizado já que há a possibilidade de permanecer 24 horas por dia a disposição dos alunos.

Este artigo descreve o trabalho que vem sendo desenvolvido com a Prof ${ }^{a}$. Elektra, um robô de bate-papo (chatterbot) baseado no chatterbot ALICE, que vem sendo utilizado como um instrumento de complementação no aprendizado de estudantes de cursos à distância.

O artigo está organizado da seguinte forma: a Seção 2 apresenta os conceitos de chatterbot e trabalhos relacionados, a Seção 3 apresenta uma rápida abordagem sobre o conceito de Educação à Distância, a seção 4 descreve o chatterbot ALICE e a linguagem AIML, a seção 5 mostra a implementação do chatterbot ALICE no ensino de Redes de Computadores, a Prof ${ }^{a}$ Elektra e a Seção 6 finaliza este artigo com as conclusões e trabalhos futuros.

\section{Chatterbot}

Um chatterbot é um programa que procura simular uma conversação, com o objetivo de levar o interlocutor a pensar que está falando com outro ser humano [Laven]. Essa possibilidade de se dar a uma máquina habilidade para interagir com o ser humano, através de uma compreensão e simulação do seu comportamento, tem sido, há muito tempo, alvo de pesquisas na área de Inteligência Artificial. Nesse contexto, foram surgindo, ao longo dos anos, diversas categorias de chatterbots são utilizados na internet: comerciais, de entretenimento, $F A Q s^{1}$, de busca, educacionais, entre outros. Riesbeck e Schank (1989) desenvolveram a teoria de Scripts em seu estudo sobre o processamento da linguagem natural

\footnotetext{
1 Frequently Asqued Questions. Em português: perguntas mais freqüentes.
} 
Dentre os chatterbots já existentes, um dos mais antigos pode ser considerado o Eliza [Eliza 1966]. Desenvolvido em 1966 pelo professor Joseph Weizenbaum no Massachussets Institute of Technology, seu objetivo é o de simular um psicanalista em uma conversa com seu paciente. Eliza espera que o usuário conte seus problemas e interage através de perguntas, estimulando o paciente a contar cada vez mais sobre seu problema, através de uma personalidade bem notável, tentando sempre agir de forma simpática e amável.

O Eliza, porém, tem algumas limitações. Não há uma memória no robô, ou seja, ela não consegue lembrar o que foi falado anteriormente. A construção de algumas respostas também pode desanimar o usuário, já que Eliza muitas vezes na tentativa de dar ao dialogo uma certa naturalidade, responde ao interlocutor usando partes da própria entrada, gerando às vezes diálogos um tanto confusos. Um exemplo dessa situação pode ser ilustrado da seguinte forma: se a entrada for, por exemplo, a frase: "I am fine now thank you", a resposta pode ser algo como: "How long have you been fine now thank $i$ ?". Quando a entrada apresenta complementos além de sua estrutura normal, o robô tende a dizer frases sem sentido.

Outro robô capaz de interagir em português, é Cybelle[Cybelle 2000], criada em 2000. Apresentando uma imagem virtual, Cybelle é construída para ser uma espécie de referência no mundo dos agentes. Além de conversar com ela, pode-se visualizar, ao mesmo tempo, informações sobre agentes virtuais. Cybelle também está integrada a um portal sobre agentes. A navegação no portal pode ser direcionada durante a própria conversa com o robô por ela mesma ou pelo usuário independentemente. Assim, se a entrada for: "O que exatamente você é?", Cybelle além de fornecer a resposta ainda disponibiliza uma página com informações sobre de sua personalidade. Se a entrada é uma pergunta sobre ALICE, por exemplo, Cybelle mostra informações a respeito e ainda solicita a opinião do usuário sobre o Chatterbot. (figura. 1) 


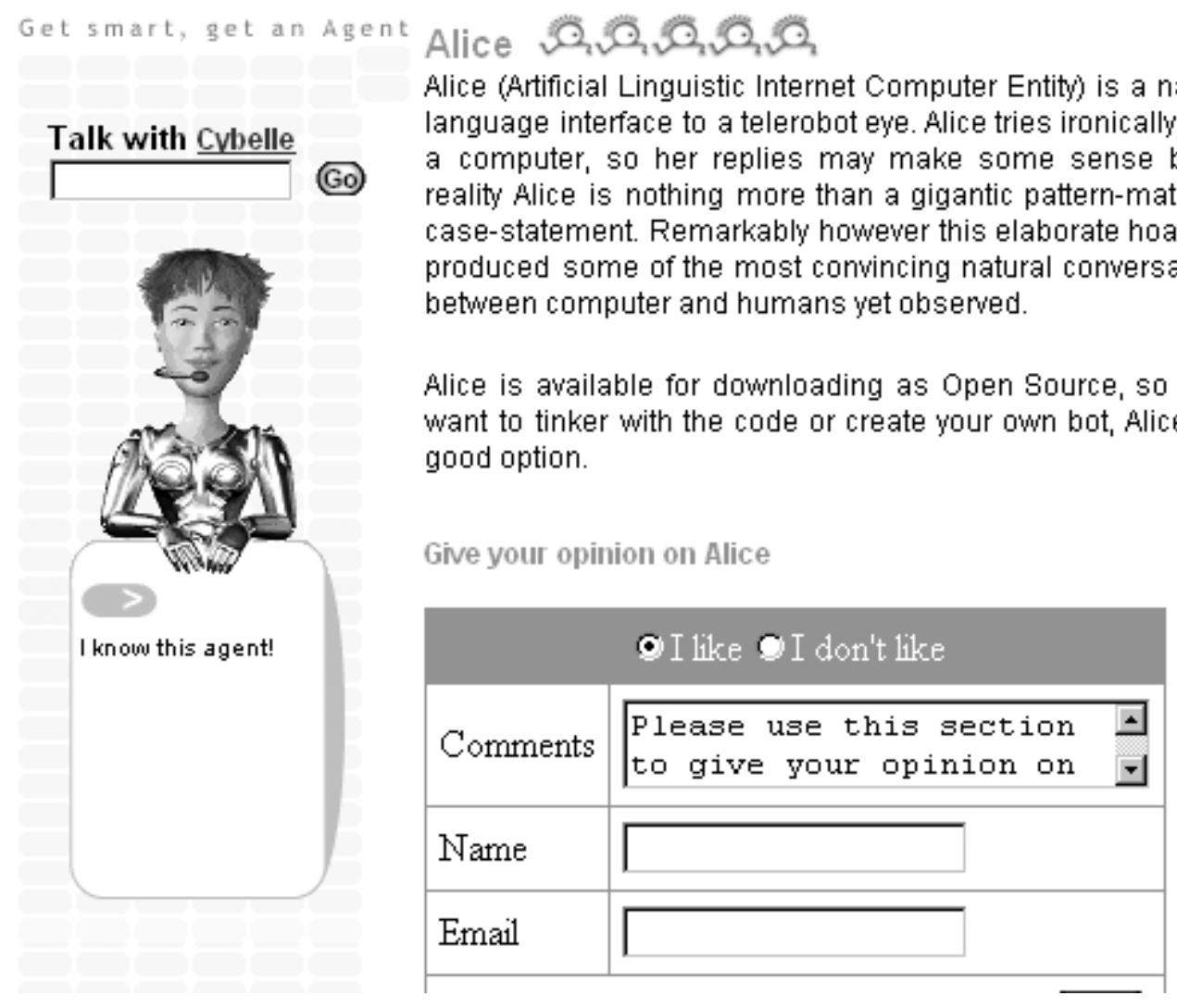

Figura 1 - Cybelle mostrando informações sobre outro Chatterbot, o ALICE, durante o diálogo

No campo dos chatterbots educacionais, existem algumas implementações, porém nenhuma delas com conteúdo temático. Junior é um robô adolescente, em português, programado para um papel educativo. Sua base de conhecimento apresenta um bom número de informações sobre conteúdos escolares[Primo 1999]. Apesar disso, Júnior não é um robô temático. Seu conteúdo abrange alguns conteúdos escolares, porém não é uma fonte de conhecimento de uma área específica.

\section{O uso de chatterbots na Educação à Distância}

A Educação à Distância (EAD) pode ser definida como uma experiência planejada de ensino e aprendizagem que faz uso de tecnologias para integrar estudantes que se encontram geograficamente distantes.

A característica básica da EAD é o estabelecimento de uma comunicação de dupla via, na medida em que professor e aluno não se encontram juntos na mesma sala requisitando, assim, meios que possibilitem a comunicação entre ambos como correspondência postal, correspondência eletrônica, telefone ou qualquer ferramenta proveniente da Internet. [Perry 1997].

A possibilidade de prover continuamente capacidade de atendimento a dúvidas dos alunos remotos que surge com o uso de chatterbots que tentam replicar o papel de um tutor virtual oferece novas possibilidades de suporte ao aluno distante. Uma das principais dificuldades deste tipo de aluno é a sensação de isolamento que experimenta pela distância no espaço e às vezes também do tempo quando seus horários de acesso ao sistema não coincidem com os do restante da turma. Um chatterbot provê a 
possibilidade de solicitar e receber explicações a qualquer momento. O processo de construir a base de conhecimento do chatterbot pode ser realizado pelo próprio aluno, pois existem ferramentas de autoria que facilitam a criação das definições a serem usadas no processamento da conversação permitindo que o aluno tenha participação ativa no processo. [Castanho 2002].

\section{ALICE}

O A.L.I.C.E (Artificial Linguistic Internet Computer Entity) [Alice 2003] é um chatterbot criado na Lehigh University por Richard S. Wallace, ativada em 1995, sendo um dos robôs mais populares da atualidade.

Sua inovação está na forma como é apresentado: além de muita documentação, apresenta uma saudação sonora ao visitante, tem um grande poder de comunicação, além de uma interface gráfica que estimula o diálogo [ALICE 1995]. Atualmente existe uma Fundação que promove a disseminação do software gratuito ALICE e da AIML (artificial intelligence markup language) usada na construção do ALICE bot. O chatterbot original, ALICE tem uma base de conhecimento constituída por centenas de fatos, citações e idéias de seu criador. Apresenta um vocabulário de mais de 5000 palavras. É programada para dar muitas informações a seu respeito e pode sugerir até que o usuário a veja cantar. Muitos outros chatterbots foram construídos usando o software do chatterbot ALICE. Para isto basta construir uma nova base de conhecimento expressa em AIML. Um exemplo de chatterbot construído em Português foi o Pixelbot desenvolvidopor André Neves na UFPE [Pixelbot 2003]. Usando as classes desenvolvidas para o Pixelbot tratar expressões em Português.

\subsection{Artificial Intelligence Markup Language (AIML)}

O AIML foi desenvolvido pela comunidade Alicebot durante o período de 1995-2000 com uma gramática proprietária também chamada AIML que formava a base para o primeiro Alicebot. Após diversas atualizações visando à padronização da gramática foi adotado o XML (Extensible Markup Language).

O AIML é uma linguagem de fácil aprendizagem e utilização [Wallace]. Ela apresenta um conjunto de $\operatorname{tags}^{2}$ e comandos simples para implementação da base de conhecimento de um chatterbot e serve para analisar as mensagens enviadas pelo usuário e decidir a forma como estas mensagens devem ser respondidas.

O AIML é baseado em padrões de entrada do usuário, conhecidos como categorias. Uma frase escrita por um usuário é comparada aos padrões descritos na linguagem e com base neste processo são selecionadas ou construídas as respostas.

As principais tags do AIML são:

$<$ aiml> incia e termina um bloco programado em AIML

conhecimento

$<$ category> identifica uma "unidade de conhecimento" na base de

$<$ pattern> identifica um padrão de mensagem simples freqüentemente utilizado por usuários

$<$ template> contém a resposta para uma mensagem do usuário

\footnotetext{
${ }^{2}$ Tags significam marcações.

V.1№ 2, Setembro, 2003
} 
Uma categoria em AIML é definida da seguinte forma:

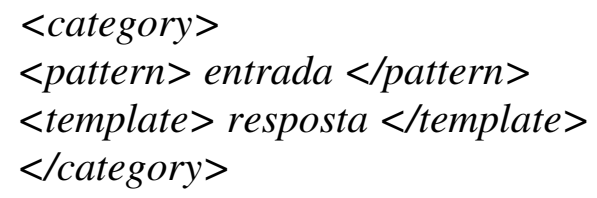

Por exemplo:

$<$ category $>$

$<$ pattern $>O I *</$ pattern $>$

$<$ template $>$ OI. TUDO BOM? </template $>$

$</$ category $>$

Neste exemplo, o padrão de entrada 'Oi” seguido de qualquer informação, terá como resposta ou possível resposta à sentença “Oi. Tudo bom?”. O caractere * representa um conjunto de caracteres.

Com a utilização de AIML, pode-se definir mais de uma resposta para um único padrão e ainda se pode especificar critérios de escolha de cada uma das respostas. Existem, ainda, mais de 20 outras tags que compõem a linguagem AIML responsáveis por fornecer a necessária desenvoltura para o Chatterbot propor uma solução à mensagem enviada.

\section{Elektra uma implementação da ALICE para no ensino de física e redes de computadores}

Com base no ALICE foi criado na UFRGS o chatterbot Prof ${ }^{a}$ Elektra. A Prof ${ }^{a}$. Elektra visou inicialmente responder perguntas sobre Física para alunos do ensino secundário que estivessem se preparando para o vestibular, sendo colocada à disposição dos internautas em meados de 2002.

Em julho de 2003 estendeu-se sua utilização para os alunos do Curso de Especialização a Distância em Informática na Educação para a disciplina Internet para Educadores, acrescentando em sua base de conhecimento dados e conceitos sobre Redes de Computadores e Internet. Para este trabalho coordenou-se um esforço entre os professores e tutores para a modelagem inicial do conhecimento buscando informar o que o robô deveria dominar, quais perguntas eram mais freqüentes e quais os assuntos que geravam maiores dúvidas entre os alunos. Para tornar a interação mais amigável foram exploradas diversas funcionalidades do AIML.

A primeira funcionalidade explorada foi à possibilidade do chatterbot escolher uma entre diversas respostas a serem apresentadas para uma mesma pergunta. Isto é feito adicionando mais de uma resposta para uma categoria de conhecimento, que é escolhida aleatoriamente no memento em que o robô é solicitado, oferecendo assim, a oportunidade de que ao ser questionado mais de uma vez sobre o mesmo assunto, o chatterbot possa apresentar uma resposta diferente da primeira. Este recurso parte do pressuposto que se o usuário do ambiente tornou o questionar o robô sobre um mesmo tópico é porque provavelmente a resposta apresentada não satisfez sua necessidade. É importante ressaltar que o robô não necessariamente apresenta uma resposta diferenciada se o assunto for persistido, visto que as respostas são escolhidas de forma 
aleatória, podendo assim a mesma resposta ser apresentada novamente. A estrutura AIML que permite respostas aleatórias é estruturada de seguinte forma no arquivo de extensão AIML do chatterbot:

$<$ aiml $>$

$<$ category $>$

$<$ pattern $>$ Palavra chave $<$ /pattern $>$

$<$ template $>$

$<$ random $>$

$<\mathrm{li}>$ Resposta $1</ \mathrm{li}>$

$<$ li $>$ Resposta $2</$ li $>$

$<$ random $>$

$</$ template $>$

$</$ category $>$

$</$ aiml $>$

Onde dentro da $\operatorname{tag}$ random $(<$ ramdom $>*<$ random $>$ ) são definidas as respostas a serem apresentadas, e cada resposta diferente deve estar dentro de uma tag li $(<\mathrm{li}>*</ \mathrm{li}>)$.

Outra funcionalidade explorada foi à adição e visualização de hiperlinks ${ }^{3}$ e imagens nas respostas. Os hiperlinks podem ser colocados em qualquer local dentro do espaço de resposta, fazendo o uso da tag a href da linguagem HTML, e as imagens podem ser exibidas usando outra tag da linguagem HTML chamada img src.

${ }^{3}$ Hiperlinks são ligações para outros documentos.

V.1№ 2, Setembro, 2003 

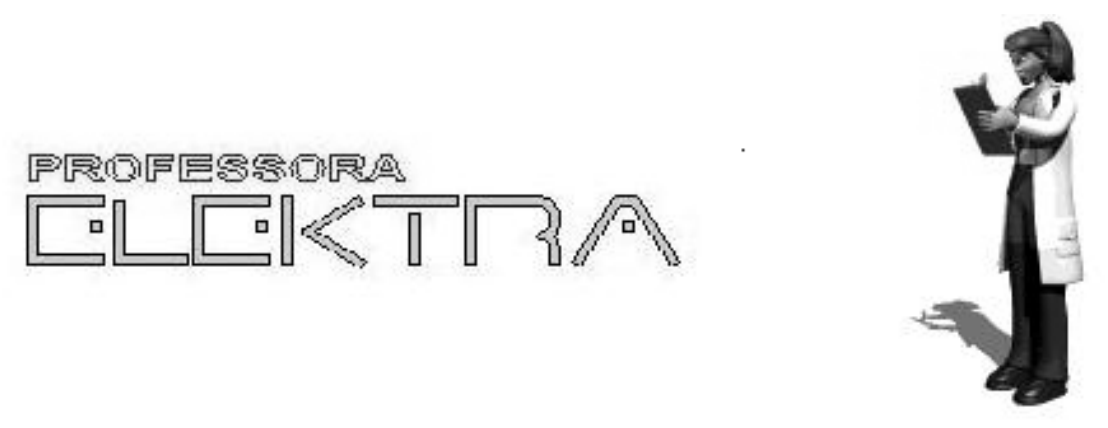

Você Disse:

Professora Elektra:

\section{Oqué intemet}

Conjunto global de redes, interliga mais de 100 paises.

Tem duas peculariedades que espantam as pessoas: nao

tem dono e nao e administrada por nenhum orgao

central.

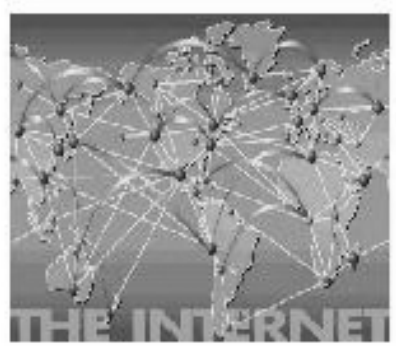

\section{Pergunte}

Você está falando com Elektra de penta3.ufrgs.br.

Elektra e'a Professora Elektra UFRGS - Brasil.

\section{Figura 2 - Exemplo de Resposta com Imagem}

O AIML também possibilita usar uma tag chamada <srai> que serve para direcionar uma entrada (pergunta), para outra estrutura que já tenha uma resposta formulada que se quer apresentar, ela direciona uma entrada (pergunta) de um pattern para um outro pattern já existente, então a procura encontra o pattern que trata a entrada que o <srai $>$ indica.

$<$ category $>$

$<$ pattern $>$ entrada $1<$ pattern $>$

$<$ template $><$ srai $>$ entrada $2<$ /srai $><$ template $>/ /$ aqui a tag srai direciona a pesquisa para uma outra categoria onde o teste padrão é "entrada2" que tem a resposta que se quer apresentar.

$<$ category $>$

$<$ category $>$

$<$ pattern $>$ entrada2 </pattern $>/ /$ ele procura por esta estrutura onde $\mathrm{o}$

paterrn foi indicado pelo $<$ srai $>$

$<$ template $>$ resposta $<$ template $>/ /$ e apresenta esta resposta $</$ category $>$ 
Outra funcionalidade interessante do AIML é a utilização da tag <that>. A palavra "that" AIML se refere a decraração anterior do chatterbot. Especificamente se este responde com um parágrafo de múltiplas sentenças, o valor do "that" e setado para a última sentença na sequiência. A seguir um exemplo de como isto pode ocorrer.

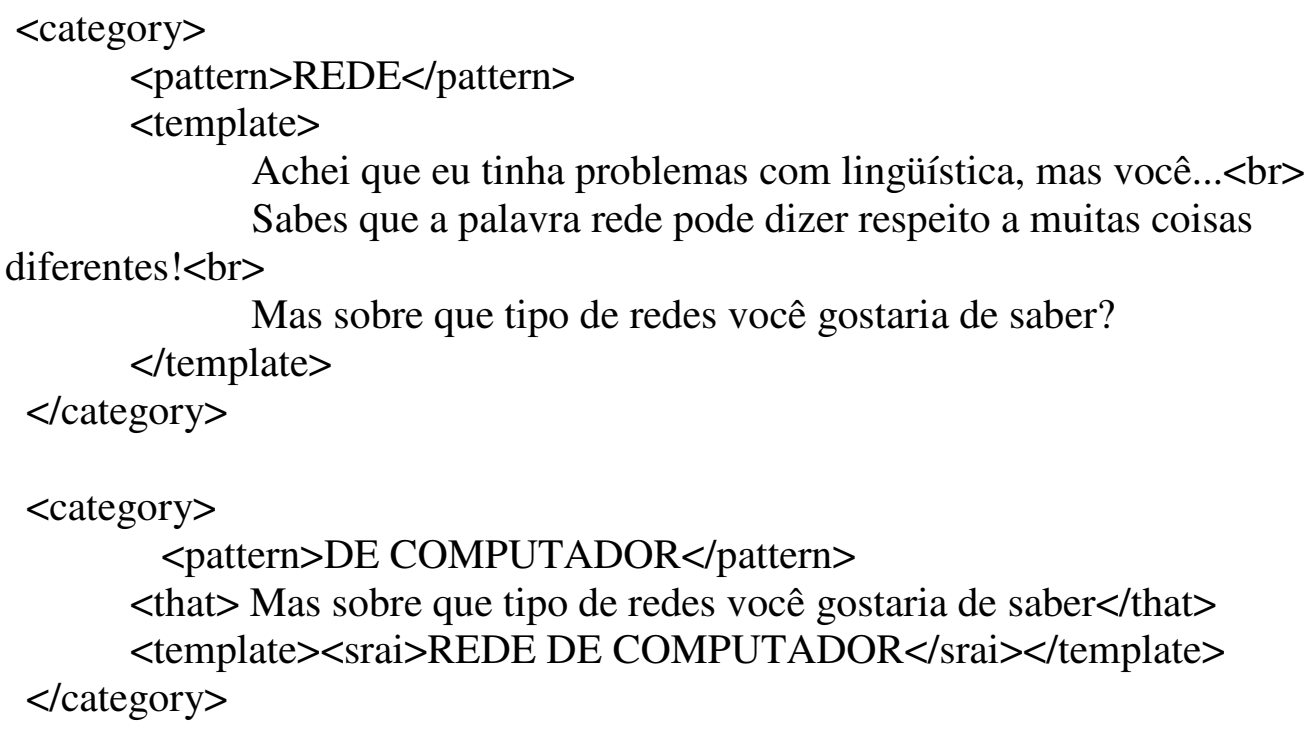

Neste caso a categoria "DE COMPUTADOR" é ati vada somente quando o aluno digita esta frase respondendo a pergunta do chatterbot: "Mas sobre que tipo de redes você gostaria de saber". Este recurso enriquece a conversação, simulando uma memória da conversa apesar de ainda limitada à última sentença.

$\mathrm{O}$ uso de respostas modeladas com as funcionalidades descritas pareceram em primeiro momento serem o suficientes, pois previa diversidade de respostas, trazia ilustrações e direcionava o usuário a tutorias e Websites dedicados ao assunto trabalhado, entretanto para avaliar a qualidade das interações, em um segundo momento, resolveu-se analisar as respostas dos alunos verificando os arquivos de $\log$ (registros) gerados pelo servidor da Prof ${ }^{a}$. Elektra nas interações dos alunos do curso de Especialização em Informática na Educação. O Program D que é o software servidor da Elektra (ALICE) gera arquivos de log com todas as conversas no padrão XML e através da análise destes logs notou-se que apesar de suas respostas serem ricas em conteúdo, a comunicação não estava adequada.

Analisando estes arquivos um dos problemas observados foi que as respostas do chatterbot apresentavam-se muito diretas e objetivas, de forma que o chatterbot não estimulava a continuação do diálogo. O segundo problema foram as diferentes formas de comunicação expressas pelos alunos nas suas interações com o chatterbot, visto que estes eram de regiões diferentes do Brasil. As perguntas sobre um mesmo tópico eram realizadas das mais diversas formas, fugindo assim da modelagem prevista e aplicada às perguntas da Prof $^{a}$. Elektra, então o robô, muitas vezes era portador do conhecimento questionado pelo aluno, mas não apresentava a resposta correspondente uma vez que não reconhecia a pergunta.

Tendo em mãos este material, iniciou-se a tarefa de correções e aperfeiçoamentos no chatterbot. A primeira etapa foi melhorar as respostas, deixando a Prof $^{\mathrm{a}}$. Elektra aparentemente mais amigável, e interessada no assunto ao qual estivesse tratando com o aluno, de forma a estimular a continuidade do dialogo. Nesta etapa, foram revisadas as respostas que o chatterbot fornecia, acrescentando assim no final de algumas das respostas perguntas e outras informações ao usuário, ou pedidos para que 
questionassem mais o chatterbot. Os resultados deste ajuste, logo foram notados. Os diálogos com o robô passaram a ter um tempo de duração maior, e a impressão de que o robô os compreendia e interessava-se pelo assunto sugerido deixou-o mais acessível.

A segunda etapa demandou maior atenção. Pois dizia respeito as diferentes formas de se perguntar a mesma coisa ao robô. Aqui através de uma análise minuciosa do log extraiu-se cada pergunta (categoria) que não estava registrada na base de conhecimento do bot. Como na maioria das vezes o robô já tinha uma resposta pronta, adicionou-se então estas perguntas a base de conhecimento do chatterbot de maneira que ele apresentasse as respostas já existentes sobre aquele assunto.

Outros aperfeiçoamentos na base de conhecimento do chatterbot poderão ser implementados com utilização de recursos mais avançados do AIML e a criação de novas categorias oriundas de novas interações dos alunos com a Prof ${ }^{a}$. Elektra.

\section{Conclusões e Trabalhos Futuros}

Com o avanço dos estudos de processamento de linguagem natural e de inteligência artificial, os robôs de conversação cada dia desenvolvem novas habilidades e são utilizados para os mais diversos fins.

O uso de robôs de conversação na educação pode ser muito vantajoso, dependendo do contexto em que o mesmo for inserido. Por imitar a realidade humana e ser de fácil manuseio, um robô permite que um visitante não se sinta constrangido ao manusear e interagir com ele. Há também a possibilidade de direcionar o interlocutor, ou seja, se o cérebro do robô conhece bons endereços com informações relevantes sobre um determinado assunto, ele pode direcionar o usuário convidando-o a visitar estes endereços que foram previamente verificados e validados pelo programador da base de conhecimento.

O chatterbot descrito apresenta um forte apelo no sentido de atiçar a curiosidade e a motivação dos alunos. Por ser uma forma diferente de assimilação de conteúdos, a Prof $^{a}$ Elektra pode incentivar o trabalho dos usuários já que exige deles uma participação muito mais ativa, onde quem comanda e direciona o diálogo é o próprio interlocutor. Porém observou-se que somente com uma extensa utilização da Prof ${ }^{a}$ Elektra pelos alunos e conseqüente análise dos registros desta utilização será possível criar novas categorias tornando a conversação mais natural e abrangente.

\section{Referências Bibliográficas}

ALICE (1995) The A. L.I.C.E Foundation http://alicebot.org, Acesso em abril de 2003

Brusil, P. J. and Collins, Will (1991) 'OSI Systems and manaement standards”. Global Communications Conference.

Castanho, Carla, Wazlawick, Raul. A Avaliação do Uso de Chatterbots no Ensino Através de uma Ferramenta de Autoria. SBIE 2002. São Leopoldo, 2002.

Cybelle (2000) http://www.agentland.com/, April

ELIZA (1966) - Weizenbaum, J. http://www-ai.ijs.si/eliza/eliza.html, Abril

Laurel, B. (1997). Interface agents: Metaphors with Character. In J. M. Bradshaw (Ed.) Software Agents p. 67- 77. Menlo Park, CA: AAAI Press/The MIT Press.

Laven, S.J. “The Simon Laven Page” http://www.simonlaven.com/, Abril 2003. 
Perry, W.; Rumble, G. (1987). "A short guide to distance education”. Cambridge: International Extension College.

Pixelbot. Projeto Virtus da UFPE http://www.virtus.ufpe.br. Acesso em Junho 2003.

Primo, A.F.T; Coelho, L R and Tamusiunas, F.R. (1999) 'O uso de chatterbots no ensino a distancia” X Simpósio Brasileiro de Informática na Educação.

Taylor, A. The AIML mini reference and primer. [http://hippie.alicebot.com/ ataylor/index.html], Abril

Turing, A.M. (1950). ‘Computing machinery and intelligence”. Mind, 59, 433-560.

Wallace R.S. "AIML Overview" http://www.pandorabots.com/pandora/pics/wallaceaimltutorial.html, Abril 2003

Weizenbaum, J. (1966) 'ELIZA: A Computer Program For the Study of Natural Communication Between Man and Machine". Communications of the ACM. Vol. 9, No. 1, pp.36-45. 